population, 245748 individuals were reported with long-term diseases. Key statistical signals are presented for several disease groups (respiratory, neoplasms, neurodegenerative, etc).

Discussion The approach presented had the following advantages:

- enabling systematic evaluations of all disease - occupational activity associations,

- high statistical power, and

- costless data acquisition.

The main drawback is its lack of direct information regarding exposure. For this reason, further work is currently performed to estimate exposure to pesticides retrospectively relying on other databases. This data mining approach will later be enriched by identifying diseases using the medicalrelated expense databases (including information on medications, biological exams, etc).

\section{OCCUPATIONAL RISKS FOR MIGRANT WORKERS IN SPAIN}

${ }^{1} \mathrm{E}$ Ronda*, ${ }^{1} \mathrm{~A}$ Cayuela, ${ }^{2} \mathrm{E}$ Koselka, ${ }^{1} \mathrm{I}$ Benazizi, ${ }^{1} \mathrm{JM}$ Martínez. ${ }^{1}$ University of Alicante, Alicante, Spain; ${ }^{2}$ Northwestern University, Evanston, USA

\subsection{6/oemed-2018-ICOHabstracts.345}

Introduction Migrant populations appear to be in general at greater risk than native populations for developing a number of diseases. However, the health and safety environments for these migrant populations are scarcely studied. The objective of this study is to compare the prevalence of risk exposures between migrant workers and Spain-native workers in Spain.

Methods Information gathered through the ongoing Platform of Longitudinal Studies of Immigrant Families (PELFI; started 2015) provides insight into the different kinds of health and safety risks that migrant and Spain-native workers experience. For the present study, migrants from Colombia, Morocco, and Ecuador, as well as Spanish controls make up a cohort of 473 workers. Data on sociodemographic variables (age, sex, and type of job) and self-reported occupational exposures were collected through face-to-face interview. Analysis of specific risks to worker health and safety focused on [1] frequency of exposure to chemical, psychosocial, and ergonomic hazards, and [2] employment arrangements. Data were analysed using STATA.

Results The overall frequency of hazardous exposure was consistently higher for migrants when compared to Spain-native workers. PELFI results show significant differences $(p<0.05)$ between migrant and Spain-native workers for occupations involving heavy lifting $(38.85 \%$ migrants; $19.35 \%$ natives $)$, high heights $(16.35 \%$; 3.30\%), repetitive movements $(81.58 \%$; $59.78 \%)$, chemical products $(50.76 \% ; 18.28 \%) ; \%)$, experiencing pain as a result of the work $(59.39 \% ; 1.57 \%)$, and standing long hours (67.68\%; 19.35\%). 26.32\% of migrant workers and $15.48 \%$ of Spain-native workers reported that their salaries were insufficient to cover unexpected expenses.

Conclusion Migrant workers in this study are more frequently exposed to occupational risks than Spain-native workers. Although limitations exist regarding participants' different perceptions of risk, results suggest that special attention must be paid to migrant workers in Spain. A promising area for future research seeks to understand the processes by which workers are differentially exposed to occupational risks.

\section{APPLICATION OF METABOLOMIC SCIENCE IN THE DETECTION OF LUNG CANCER: A CASE-CONTROL} STUDY

${ }^{1,2}$ Chi-Hsiang Huang, ${ }^{3}$ Chian Zeng* ${ }^{3,4}$ Che-Jui Chang, ${ }^{3} H s i n-Y i$ Peng, ${ }^{1,2}$ Yi-Chia Wang, ${ }^{3,5} \mathrm{Hsiao}-Y u$ Yang ${ }^{*}$. Department of Anesthesiology, National Taiwan University College of Medicine, Taipei, Taiwan; ${ }^{2}$ Department of Anesthesiology, National Taiwan University Hospital, Taipei, Taiwan; 3 Institute of Occupational Medicine and Industrial Hygiene, National Taiwan University College of Public Health, Taipei, Taiwan; ${ }^{4}$ Department of Family Medicine, National Taiwan University Hospital, Taipei, Taiwan; ${ }^{5}$ Department of Public Health, National Taiwan University College of Public Health, Taipei, Taiwan

\subsection{6/oemed-2018-ICOHabstracts.346}

Introduction Lung cancer is the leading cause of cancer death in the world. The challenge of screening for early stage lung cancer is still unresolved. The exploration of metabolites in breathe using sensor array technique may become a powerful screening tool to solve the problem.

Methods We conducted a prospective study to enrol cases of lung cancer and controls who received surgery for gall bladder stone, hernia, hemorrhoid resection, and thoracoscopic surgery in the same hospital between July 2016 and June 2017. The alveolar air of subjects were collected under the guidance of mainstream carbon dioxide analyzer. An electronic nose composed of 32 carbon nanotubes sensors was used to measure the VOCs of the alveolar air. The diagnostic accuracy was analysed by linear discriminant analysis (LDA) using the pathological reports as the reference standard.

Results After excluding 2 subjects with technical problems in sampling, 12 subjects with cancers in other sites, benign lung tumour, or metastatic lung cancer, 5 subjects received chemotherapy, 5 subjects with diabetes, 2 subjects with asthma, and 2 subjects with chronic obstructive pulmonary disease, a total of 17 cases and 105 controls were used in the final analysis. We randomly split the data into $80 \%$ for model building (training set) and 20\% for validation (test set). By LDA, the accuracy, sensitivity, specificity, false positive rate, false negative rate, and ROC-AUC were 96.9\%, 75.0\%, 100.0\%, 0\%, 25\%, and 0.98 (95\% CI: 0.96 to 1.00 ) in the training set, and $84.0 \%, 80.0 \%, 85.0 \%, 15.0 \%, 20.0 \%$ and 0.84 (95\% CI: 0.62 to 1.00$)$ in the test set.

Conclusion The use of sensor array technique to explore the metabolites in breathe may become a powerful tool in the screening for lung cancer. Standardised procedures to eliminate confounding factors are warranted before clinical application.

\section{DIAGNOSIS OF 1,561 COMPENSATED CASES FOR OVERWORK-RELATED CEREBROVASCULAR/ CARDIOVASCULAR DISEASES (CCVDS) KNOWN AS 'KAROSHI' IN JAPAN, 2010-2014}

${ }^{1} \mathrm{~T}$ Yoshikawa, ${ }^{1} \mathrm{~T}$ Sasaki, ' $\mathrm{S}$ Matsumoto, 1,2 $\mathrm{T}$ Yamauchi, ${ }^{1,3} \mathrm{~K}$ Kayashima, ${ }^{1} \mathrm{~T}$ Kubo, 'S Umezaki, 'M Takahashi. 'Research Centre of Overwork-Related Disorders (RECORDS), National Institute of Occupational Safety and Health (JNIOSH), Japan; ${ }^{2}$ Department of Public Health and Environmental Medicine, The Jikei University School of Medicine, Tokyo, Japan; ${ }^{3}$ Bodhi Health Care Support Ltd., Tokyo, Japan

\subsection{6/oemed-2018-ICOHabstracts.347}

Introduction Overwork-related health disorders including cerebrovascular/cardiovascular diseases (CCVDs) and mental disorders, known as 'Karoshi', have been the keen occupational health issue. Main criteria applied for compensation of overwork-related CCVDs in Japan were (1) extraordinary events, 
(2) excessive working hours in 1 week and (3) long working hours over 100 extra hours in 1 month or over average 80 extra hours in 2-6 months. The present study examined diagnosis of compensated cases as overwork-related CCVDs in Japan.

Methods The investigation report for the occupational accident related to CCVDs prepared by 321 labour standards inspection offices all over Japan from January 2010 to March 2015 were collected to JNIOSH-RECORDS. Total 1561 cases which met these criteria were examined from the viewpoint of the diagnosis-related in focusing on sex, age, size of the industry. This study was supported by the research fund of MHLW (No. 150903-01).

Results The majority (96\%) of all compensated CCVDs cases was male. Fatal cases accounted for $39.2 \%$. A mean age at the onset of CCVDs was 49.3 years old. Intracerebral haemorrhage $(\mathrm{ICH})$ was the highest $(28.6 \%)$ in the proportion of diagnosis related group, following subarachnoid haemorrhage (SAH, 18.5\%), cerebral infarction (CI, 14.6\%), hypertensive encephalopathy $(\mathrm{HE}, 0.3 \%)$ in a cerebrovascular disease group. In a cardiovascular disease group, myocardial infarction (MI, $17.1 \%$ ) was the highest, following cardiac arrest (including sudden cardiac death) (CA, 14.3\%), dissecting aortic aneurysm (DAA, 5.2\%), angina pectoris (AP, 1.2\%). By classifying the industry size, CCVD cases at the workplace hiring less than 50 workers was over half of all cases (52.2\%).

Discussion Focusing on cerebrovascular diseases accounting for $60 \%$ is useful for examining preventive measures. Characteristics of diagnosis contribute to discuss directions for preventing overwork-related CCVDs. It should be paid attention to preventive measures in small sized enterprises that occupational health services are difficult to reach.

\section{LUNG CANCER AND OCCUPATIONAL SOCIAL STATUS: THE SYNERGY STUDY}

\begin{abstract}
${ }^{1}$ Thomas Behrens*, ${ }^{1}$ Jan Hovanec, ${ }^{2}$ Jack Siemiatycki, ${ }^{3}$ David I Conway, ${ }^{4}$ Ann Olsson, ${ }^{5}$ Hans Kromhout, ${ }^{5}$ Roel Vermeulen, ${ }^{4}$ Kurt Straif, ${ }^{6}$ Karl-Heinz Jöckel, ${ }^{1}$ Thomas Brüning. ${ }^{1}$ Institute for Prevention and Occupational Medicine of the German Social Accident Insurance (IPA), Institute of the Ruhr-Universität Bochum, Bochum, Germany; ${ }^{2}$ University of Montreal, Hospital Research Centre (CRCHUM) and School of Public Health, Montreal, Canada; ${ }^{3}$ Dental School, College of Medicine Veterinary and Life Sciences, University of Glasgow, Glasgow G2 3JZ, UK; ${ }^{4}$ International Agency for Research on Cancer (IARC), Lyon, France; ${ }^{5}$ Environmental Epidemiology Division, Institute for Risk Assessment Sciences, Utrecht, The Netherlands; ${ }^{6}$ Institute for Medical Informatics, Biometry and Epidemiology, University Hospital Essen, Essen, Germany
\end{abstract}

\subsection{6/oemed-2018-ICOHabstracts.348}

Introduction Several studies associated low socioeconomic status (SES) with lung cancer. However, many were not able to consider smoking behaviour appropriately. We took advantage of the international SYNERGY study of pooled case-control studies with detailed information of smoking habits and the occupational history to study the association between lung cancer and occupationally derived SES.

Methods Twelve case-control studies from Europe and Canada were included. We estimated SES based on the subjects' complete occupational histories using the International Socio-Economic Index of Occupational Status (ISEI) and the European Socio-economic Classification (ESeC). ISEI was categorised into four equidistant categories comprising the same number of codes and, secondly, according to quartiles of the sex-specific score distribution among control subjects. We calculated odds ratios (OR) and 95\% confidence intervals (CI) by unconditional logistic regression, adjusting for age, study, and smoking behaviour, and stratified by sex. Subgroup analyses by lung cancer histological subtype, study region, birth cohort, education, and occupational exposure to known lung carcinogens were also carried out.

Result We included 17021 cases and 20885 control subjects into the final analysis. There was a strongly elevated association of lung cancer with low SES in the analysis adjusted for age and study. Adjustment for smoking attenuated the associations, however, a social gradient with lung cancer persisted. Comparing the lowest vs highest SES category in men yielded: ISEI $\mathrm{OR}=1.84(95 \% \mathrm{CI}: 1.61$ to 2.09$)$ and $\mathrm{ESeC} \mathrm{OR}=1.53$ (95\% CI: 1.44 to 1.63$)$. ORs for women were slightly lower: ISEI OR $=1.54(95 \% \mathrm{CI}: 1.20$ to 1.98$)$ and $\mathrm{ESeC} \mathrm{OR}=1.34$ (95\% CI: 1.19 to 1.52 ).

Discussion Low SES remained an independent risk factor for lung cancer even after controlling for smoking habits.

\section{IT IS THE RESIDENCY IMPORTANT FOR WORKING CONDITIONS? A CROSS-SECTIONAL STUDY ON MIGRANT DOMESTIC WORKERS IN ARGENTINA} ${ }^{1,2}$ María Fernanda Bauleo*,${ }^{2,3}$ Frank van Dijk, ${ }^{2}$ Katja Radon. ${ }^{1}$ MunBaus Consultants,
Argentina; ${ }^{2}$ Center for International Health Ludwig-Maximilians-Universität, University
Hospital Munich, Germany; ${ }^{3}$ Foundation Learning and Developing Occupational Health
(LDOH), Leiden, The Netherlands

\subsection{6/oemed-2018-ICOHabstracts.349}

Introduction Domestic workers form an important group of migrant workers throughout the world. They offer vital services to the receiving countries. In Argentina legal framework exists to employ domestic workers, but migrants from another country need to obtain a legal residency status. The aim of this study was to compare working and employment conditions of migrant domestic workers without residency status to domestic workers with an Argentinian passport or with a residency status. Additionally, risk factors for poor health should be identified.

Methods In this cross-sectional study, 201 female domestic workers (response 94\%) answered a written questionnaire. The European Working Conditions Survey was adapted to the specific work situation. The General Health Questionnaire (GHQ-12) and one general health item were used to assess health.

Results Countries of origin included Paraguay (87\%), Peru $(8 \%)$ and Bolivia (5\%). Migrant workers without residency status (35\%) were younger, had lower education and a shorter length of service than migrants with residency status. They were more likely to work informally $(94 \%$ vs $65 \%$; $\mathrm{p}<0.001)$, more than 48 hours/week ( $58 \%$ vs $37 \% ; \mathrm{p}=0.02)$, to be exposed to violence or bullying at the workplace $(27 \%$ vs $15 \% ; p=0.05)$ and to take care of elderly $(67 \%$ vs $30 \%$; $\mathrm{p}<0.001)$. Differences remained after controlling for age and education. For all domestic workers, violence or mobbing was the main predictor for poor mental health (prevalence 12\%; OR 4.7 ; 95\% CI: 1.6 to 13.6 ) and poor/fair general health (23\%; OR 6.5; 2.7-15.4). Workers with a higher educational level and workers of 30-39 year are at high risk of poor mental health, informal workers are at low risk.

Discussion Domestic workers as an important pull factor in international migration may result in precarious employment especially for workers without a legal residency status. Violence or mobbing was the main predictor for poor health. 